\title{
OUTBURST PHENOMENA IN X-RAY BINARIES
}

\author{
Y. TANAKA \\ Institute of Space and Astronautical Science \\ 3-1-1 Yoshinodai, Sagamihara, Kanagawa-ken 229, Japan
}

ABSTRACT. Three distinctly different types of outburst phenomenon in Xray binaries are discussed: (1) the so-called type 1 burst which is a thermonuclear flash of matter accreted on the surface of neutron stars, (2) the so-called type 2 burst which is a burst-like X-ray emission caused by a spasmodic accretion, and (3) the nova-like outburst which is triggered by a sudden commencement of accretion.

\section{Type 1 X-Ray Bursts .}

The so-called type 1 burst is a sudden increase of X-ray intensity with a rise time of the order of 1 second followed by a gradual decay on a time scale of $\sim 10$ seconds to several tens of second. An example of typical type 1 burst is shown in Fig. 1. The peak luminosity is typically $\sim 10^{38} \mathrm{ergs} / \mathrm{sec}$. This type of X-ray burst has so far been detected from $\sim 35$ low-mass $X$-ray binaries of which the compact objects are neutron stars. For a comprehensive review, see Lewin and Joss (1983).

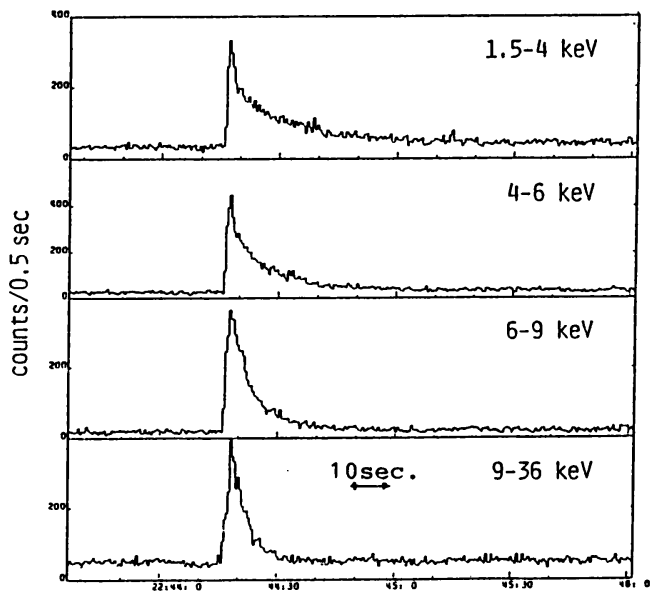

Fig. 1. A type 1 burst from X1636-53.

Important characteristics of type 1 bursts are that the spectrum is of blackbody nature, and that the color temperature decreases as burst decays, while the "apparent" blackbody radius remains constant at $\sim 10$ $\mathrm{km}$, close to the radius of neutron stars theoretically expected. These features indicate that the neutron star envelope is once quickly heated up and then gradually cools down by blackbody radiation.

Type 1 burst is convincingly interpreted as a thermonuclear flash 
of helium which is accumulated as a product of steady nuclear burning of accreted hydrogen on the surface of a weakly magnetized neutron star. Extensive studies of type 1 bursts have been made from various satellites, in particular SAS-3, Hakucho and Tenma. One of the most important outcome is that the type 1 burst provides us with a possible method for determining the mass and radius of neutron stars, as discussed in the next section.

\section{Mass and Radius of Neutron Stars}

The burst peak luminosity varies from burst to burst. However, some energetic bursts show evidences that the peak luminosity saturates. For example, Fig. 2 displays the bolometric flux, color temperature, and the apparent blackbody radius (derived assuming a $10 \mathrm{kpc}$ distance) as functions of time, respectively, for three bursts from X1636-53 observed from Tenma (Inoue et al. 1984). These cases are fully consistent with that the luminosity reached the Eddington limit, consequently resulting in an expansion of the photosphere as shown by an increase of the apparent blackbody radius in the initial phase. Temperature drops during the photospheric expansion, and then rises to the maximum value when the photosphere settles back to the neutron star surface.
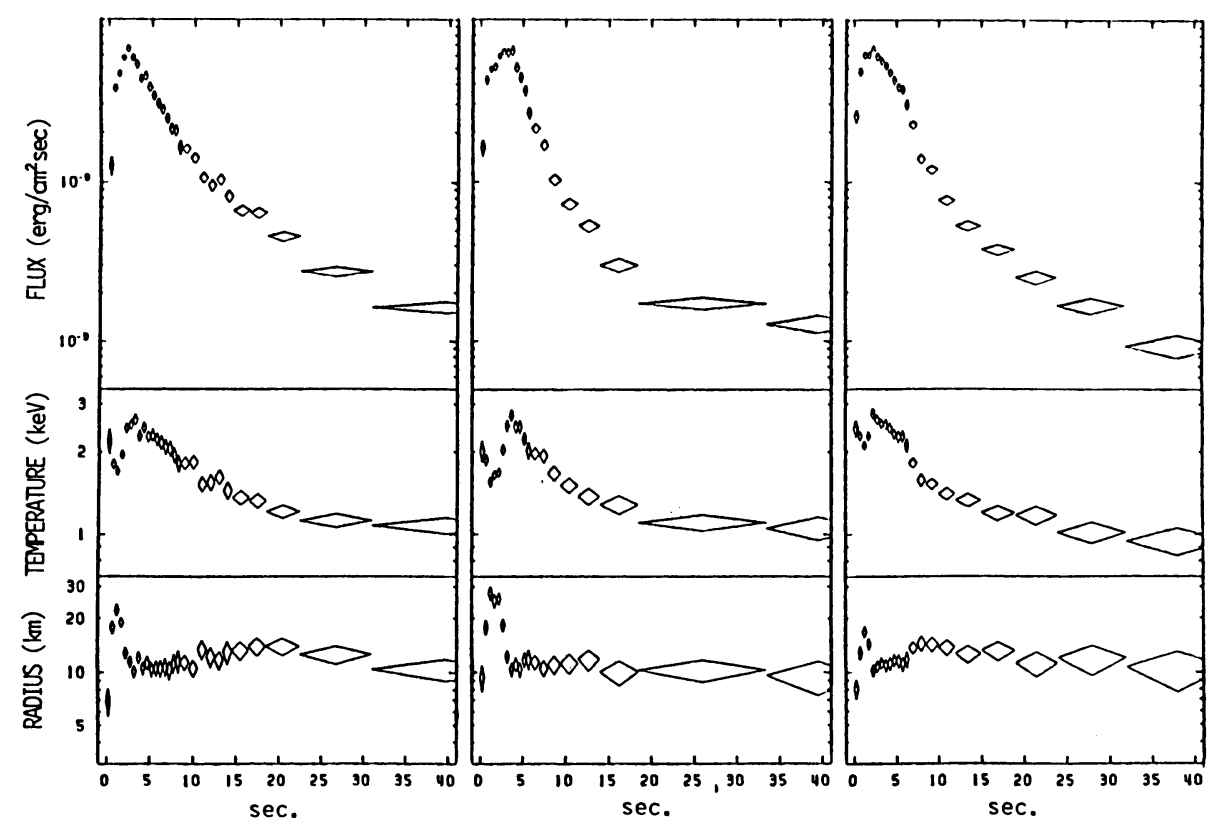

Fig. 2. Three type 1 bursts of the highest peak flux from X1636-53. Bolometric flux, color temperature, and the apparent blackbody radius are shown against time, respectively.

Based on this interpretation, the following relation holds: 


$$
\sigma \mathrm{T}_{\mathbf{e}}(\mathrm{r})^{4}=\operatorname{cGM}\left[1-\left(2 \mathrm{GM} / \mathrm{c}^{2} \mathrm{r}\right)\right]^{3 / 2} / \kappa(1+\mathrm{X}) \mathrm{r}^{2},
$$

where $r$ is the radius of the photosphere, $T_{e}(r)$ the effective temperature, $\kappa(1+X)$ the electron scattering opacity, and $X$ the hydrogen mass fraction. There is a good observational evidence to show that the highest peak luminosity is the Eddington limit for a helium-rich envelope where nuclear shell flash takes place $(X=0)$ (Inoue 1986). Thus, we obtain a relation between $R$ and $M$, if we know $T_{e}$, max when the photosphere shrinks back ( $r$ becomes practically equal to the neutron star radius $R$ ). Here, it is to be noted that the measured temperature is the color temperature $T_{c}$ and not the effective temperature $T_{e}$. For converting $\mathrm{T}_{\mathbf{c}}$ to $\mathrm{T}_{\mathbf{e}}$, we can rely on the results of theoretical calculations (e.g., London et al. 1986; Ebisuzaki 1987). Thus, Eq. 1 gives a relation between $M$ and $R$, expressed by a locus on the mass-radius plane, as shown in Fig. 3. If another independent relation between $M$ and $R$ is obtained, the mass and radius of the neutron star can be determined.

Spectroscoy of burst emission has a potential to fix another $M$ vs. $R$ relation. On the surface of a neutron star, very significant gravitational effects are expected. For instance, the energies of X-ray photons will be gravitationally redshifted by a factor

$$
(1+Z)^{-1}=\left[1-\left(2 G M / c^{2} R\right)\right]^{1 / 2} .
$$

Therefore, if we find this effect and determine the redshift factor from the $X$-ray spectrum during burst, the ratio $M / R$ is obtained from Eq. 2 .

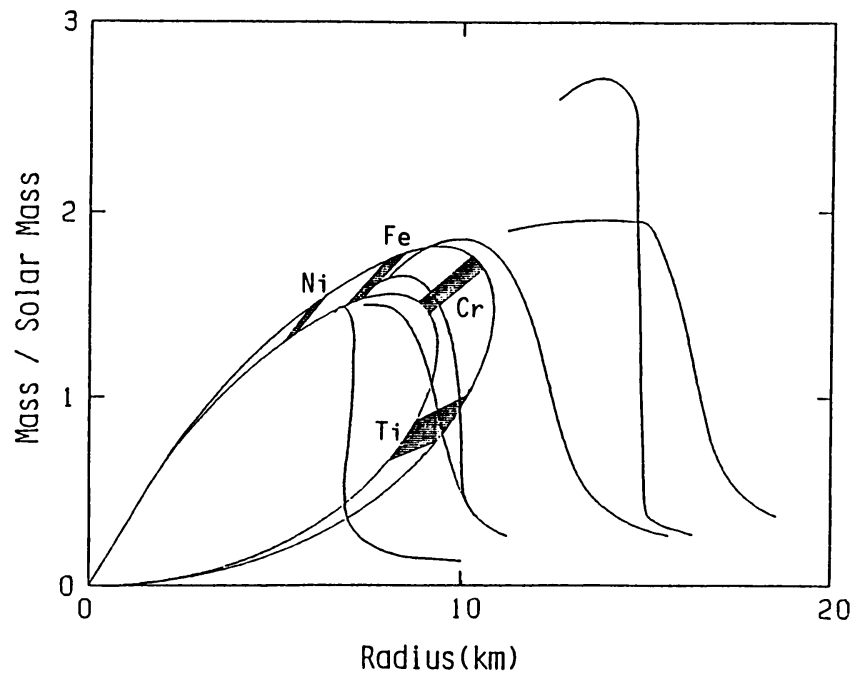

Fig. 3 .

The error domains for the mass and radius of the neutron star of X1608-52, determined for four candidate elements possibly responsible for the 4.1keV absorption line.

Theoretical models for several different equations of state of neutron stars are also shown.

In fact, significant features which look like a redshifted absorption line were discovered in some bursts from at least three low-mass X-ray binaries (Waki et al. 1984; Nakamura et al. 1988; Magnier et al. 1989), as shown in Fig. 4. A remarkable fact is that these absorption lines are always found at $4.1 \mathrm{keV}$ for all the cases, regardless of the 
$\times 1636-53$
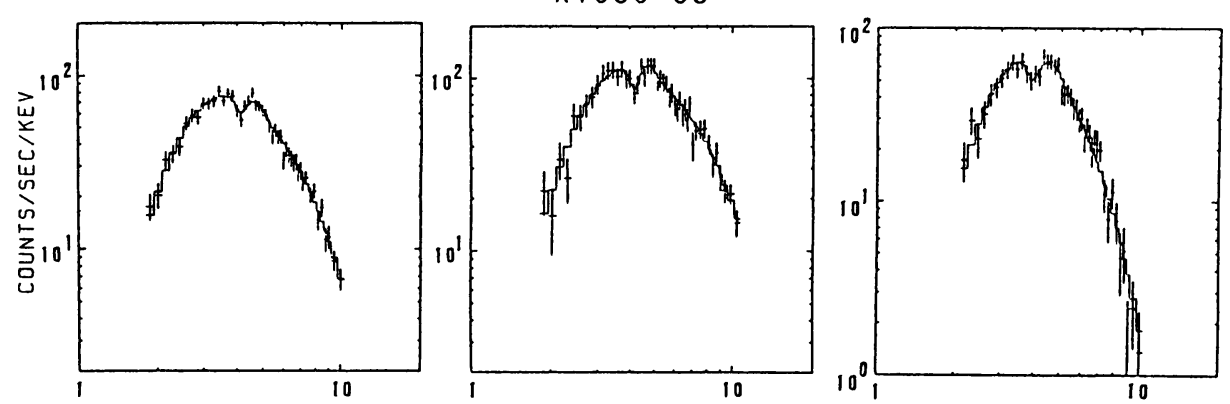

$\times 1608-52$
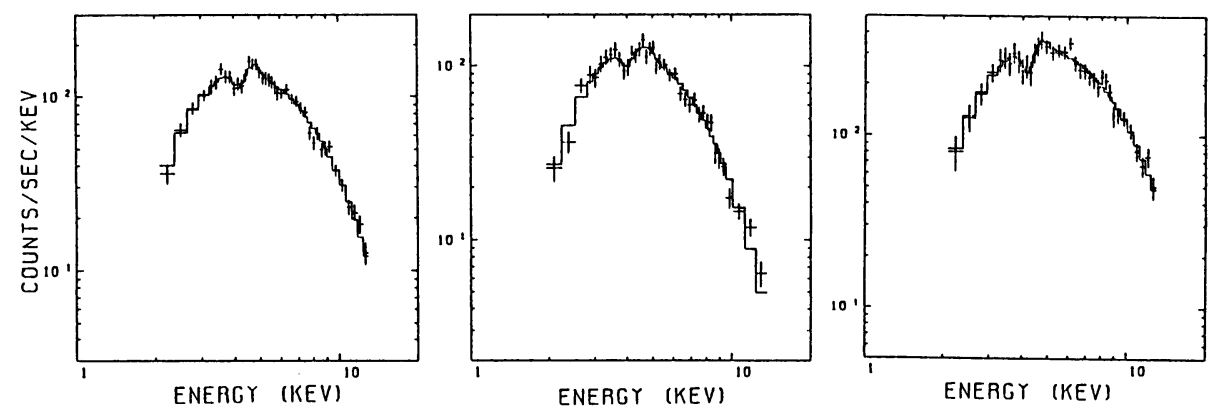

Fig. 4. Observed absorption lines in several bursts from X1636-53 and $\mathrm{X} 1608-52$.

sources. If these are indeed absorption lines by a heavy element in the neutron star envelope, one can consider four possible elements: nickel, iron, chromium and titanium produced by the nuclear flash or iron in the accreting matter. These candidate elements respectively yield different redshift factors, and hence different sets of mass and radius, as shown hatched in Fig. 3. At present, however, the element responsible for the absorption is not yet identified. Although we have to wait for future investigations of this absorption phenomenon, the observed result already excludes some of the equations of state of neutron stars (Baym and Pethick 1979), as seen in Fig. 3.

\section{Type 2 X-Ray Bursts}

Unlike type 1 bursts, type 2 bursts are those which do not show cooling with time: The burst profiles at different energies appear essentially the same. The origin of type 2 bursts is different from thermonuclear flash, but is considered to be an intermittent gravitaional energy release caused by a spasmodic accretion.

The most remarkable case is X1730-335, the Rapid Burster (Lewin and Joss 1983, and references therein) which generates a series of rapidly repetitive type 2 bursts, as shown in Fig. 5. The Rapid Burster 

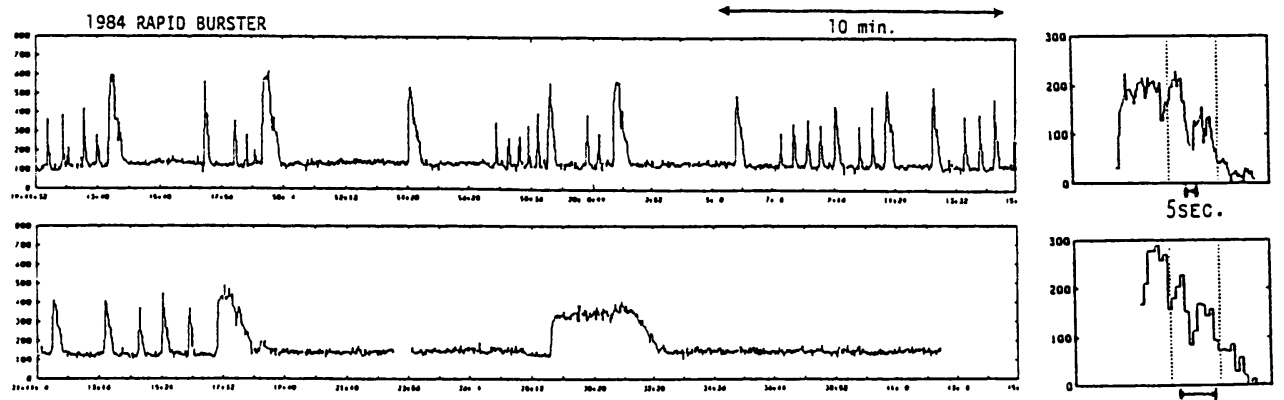

1983 RAPIO BURSTER
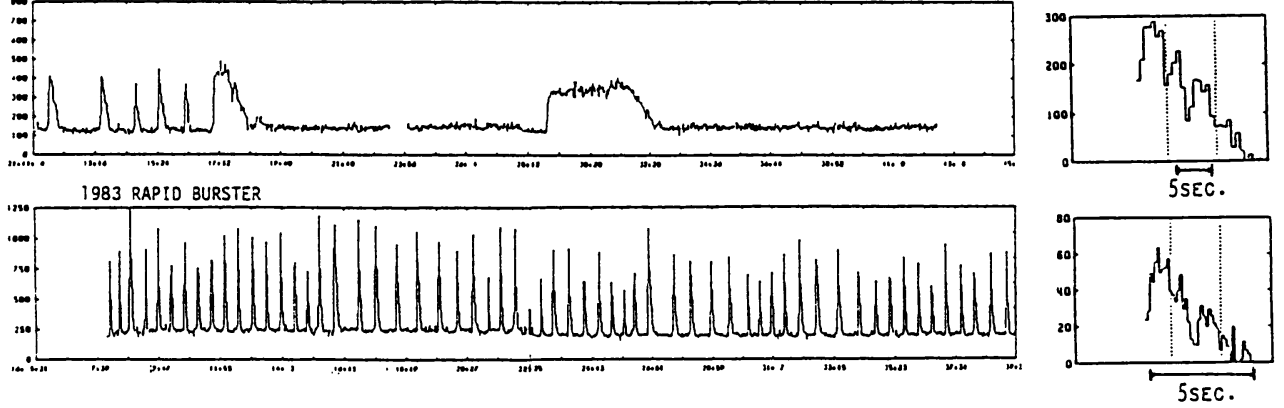

Fig. 5. Various patterns of the type 2 bursts from the Rapid Burster (left panel), and those with three different lengths (right panel), showing the time-scale invariant decay structure.

occsionally produces type 1 bursts as well. The spectrum of type 2 bursts from the Rapid Burster is approximately a blackbody spectrum, but the blackbody temperature changes very little throughout a burst (no cooling). This means that the projected area of the emission region changes according to the intensity change during a burst.

The most striking property of the bursts from the Rapid Burster is an approximately linear relation between the burst size (total energy emitted in a burst) and the waiting time to the next burst: the larger the burst size, the longer it waits till the next burst. This behavior is as thouth there exists a reservoir of mass. Suppose the reservoir is steadily supplied with mass at a fixed rate. When the reservoir is filled up, it releases an amount of mass, producing a burst. Then, the system waits until the reservoir is filled up again, to be ready for the next burst. Hence, the waiting time is proportional to the amount of mass released in the preceding burst.

The Rapid Burster exhibits many more distinct properties. For instance, each burst from the Rapid Burster has a well defined structure in the decay portion (see Fig. 5). A striking feature is that the structure is essentially identical for all bursts of various lengths, if scaled according to the burst length (Tawara et al. 1985). However, the physics of these outstanding unique properties are not yet understood.

Other than the Rapid Burster, Cir X-1 is known to produce sometimes a series of type 2 bursts (Tanaka 1986).

\section{Transient Outbursts}

There exist many transient sources in our galaxy which show up with an $\mathrm{X}$-ray outburst. $\mathrm{X}$-ray outburst is considered to be caused by a sudden 
commencement of accretion flow. In the following sections, two different classes of transient sources, the massive companion systems and the lowmass companion systems, are discussed. For a review of the earlier observational results of X-ray transients, see White et al. (1984).

\subsection{Massive Companion Systems.}

All transient sources with massive companions detected so far are X-ray pulsars. As many as ten transient $X$-ray pulsars have so far been found, nearly one third of all X-ray pulsars known to date (Nagase 1989). From the rate of discoveries, we suspect that still many more transient $X$-ray pulsars exist in our galaxy.

The optical counterparts of these transient X-ray pulsars are of ten identified with Be stars. Generally, the orbit of the neutron star in these systems is highly eccentric. Mass accretion will take place when the neutron star passes near the periastron either causing a Roche lobe overflow or accreting stellar wind. Sometimes, if the orbit is inclined with respect to the rotation axis of the massive companion, accretion will occur twice an orbit, when crossing the plane of stellar wind.

\subsection{Low-Mass Companion Systems.}

The mechanism of transient accretion in the low-mass binary systems seems entirely different from that in the massive binary systems. In the low-mass binaries, a transient accretion is considered to be triggered by a certain instability (possibly similar in part to the case of dwarf novae). However, the mechanism is far from being understood at present. The X-ray light curve exhibits a rapid rise followed by a gradual decay, representing a steady decrease of the accretion rate. Rapid brightening of an X-ray transient, or "X-ray novae", is often associated with an optical/UV brightening and also a radio outburst.

These transients are very important objects, because they allow us to study the accretion phenomena over a very wide range of accretion rate from near- or possibly super-critical rate down to lower rates by many orders of magnitude. Of particular interests are those transient sources of which the compact objects are suspected to be black holes.

\subsubsection{Transient Black Hole Candidates.}

In the last four years, the Ginga All Sky Monitor discovered three extremely bright X-ray transients: GS2000+25 (Tsunemi et al. 1989), GS2023+33 (Makino 1989), and GS1124-68 (Makino 1991) also discovered simultaneously by GRANAT (Lund and Brandt 1991). The optical counterparts of these transients were identified with very faint stars which underwent a dramatic brightening by several V-magnitudes, hence they are all low-mass stars. As discussed below, these sources are believed to be black hole candidates.

The X-ray light curves of these three transients are shown in Fig. 6 . The X-ray light curve of a black hole candidate A0620-00 (Nova Mon 1975) (Elvis et al. 1975; Kaluzienski et al. 1977) is also shown for comparison. These light curves are strikingly similar to each other. 


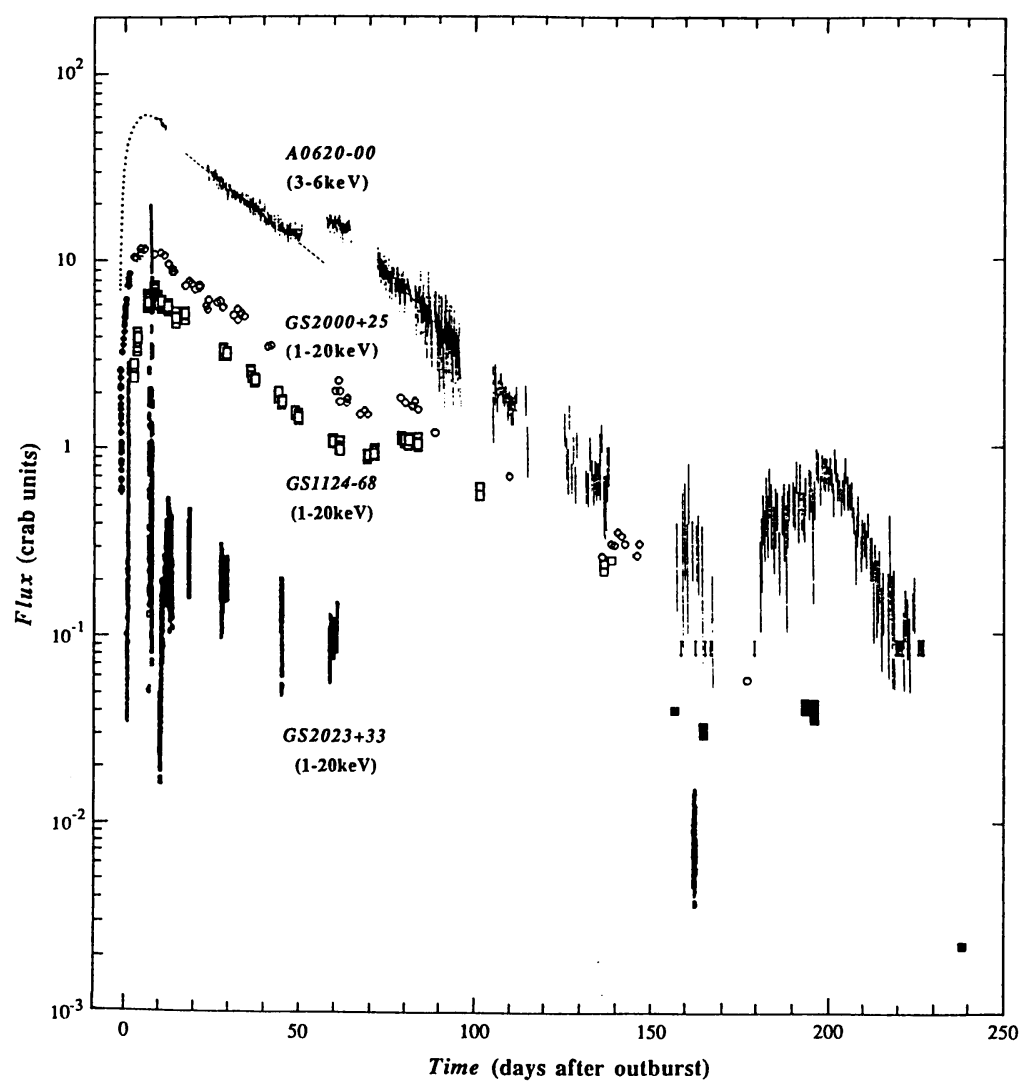

Fig. 6. X-ray light curves of three bright transients, GS2000+25, GS2023+33, and GS1124-68, and of A0620-00 for comparison.

Peak luminosities were all as high as $10^{38} \mathrm{ergs} / \mathrm{sec}$ or even higher. They all decay exponentially with essentially the same e-folding time of $\sim 40$ days. Although what governs the decay time is unknown, this result seems to indicate the similarity among their systems. Also, exponential decay implies a relation of the form $\dot{M}=-M / \tau$ to hold. This suggests that some reservoir of mass exists and the rate of accretion flow therefrom is uniquely determined by the amount of mass remaining in the reservoir.

Furthermore, at least three sources, A0620-00, GS2000+25, and GS1124-68, show a secondary increase around 60 - 70 days after the onset of outburst, and at least two of them, A0620-00 and GS1124-68, show a tertiary increase around $\sim 170$ days after the onset. For GS2023+33, the presence of these stepwise increases is inconclusive due to the lack of continued observations. These outstanding characteristics must provide important hints for the understanding of the accretion phenomna.

Here, we shall summarize the X-ray properties of the current black hole candidates in our galaxy. Among several black hole candidates, A0620-00 and Cyg X-1 are the best two, because the mass lower limits of their compact objects most certainly exceed 3Me (McClintock 1990), the 
theoretical upper limit for a neutron star. A0620-00 and Cyg X-1 respectively exhibit distinct characteristics which are very different from those of the neutron star low-mass X-ray binaries. While genuine black hole signatures are not identified as yet, those sources which show qualitatively the same X-ray properties as those of either A0620-00 or Cyg X-1 have been considered as black hole candidates.

As summarized in Table 1, A0620-00 shows a distinct energy spectrum composed of an ultrasoft component, much softer than the spectra of the neutron star low-mass X-ray binaries, and a hard power-law tail. On the other hand, Cyg X-1 shows, for most of the time, a spectrum of approximately a single power-law form, and exhibits violent and chaotic fluctuations on all time scales down to millisecond ("flickering"). These characteristics are shown in Fig. 7. It is worth noting that Cyg $\mathrm{X}-1$ sometimes, though seldom, showed an ultrasoft spectrum with a hard tail just like the spectrum of A0620-00. Therefore, the two distinctly different properties of A0620-00 and Cyg X-1 are considered to represent two states that a black hole source can assume.

Table 1. X-ray properties of the black hole candidates

\begin{tabular}{lll}
\hline Spectrum & Time Variation & Sources \\
\hline $\begin{array}{ll}\text { Hard-state } \\
\text { Approximately }\end{array}$ & Flickering & \\
a single power-law & & Cyg X-1* \\
& & GX339-4* \\
& & GS2023+33 \\
Ultrasoft-state & GS1124-68* \\
Ultrasoft & GS1826-24 \\
+ hard tail & & \\
& & A0620-00 \\
& & Cyg X-1* \\
& & GX339-4* \\
& & LMC X-3 \\
& & LMC X-1 \\
& & GS2000+25 \\
& & GS1124-68* \\
\hline
\end{tabular}

* Occasionally switches between the hard- and ultrasoft-states.

In fact, GS2000+25 closely resembles A0620-00, whereas GS2023+33 is very similar to Cyg X-1 in the spectral and temporal behaviors. GS1124-68 was initially very similar to A0620-00 in the spectral shape, but switched to another state after more than 100 days (Cyg X-1 type: a single powerlaw spectrum with flickering). Based on more detailed examinations of $\mathrm{X}$-ray properties, we consider these transients to be black hole candidates. For more detail, see papers by Tanaka $(1989,1991)$.

\subsubsection{How Many Black Hole Binaries in Our Galaxy?}

So far, only two black hole candidates, Cyg X-1 and GX339-4, are known to be persistent $X-r a y$ sources (though the latter is highly variable), 

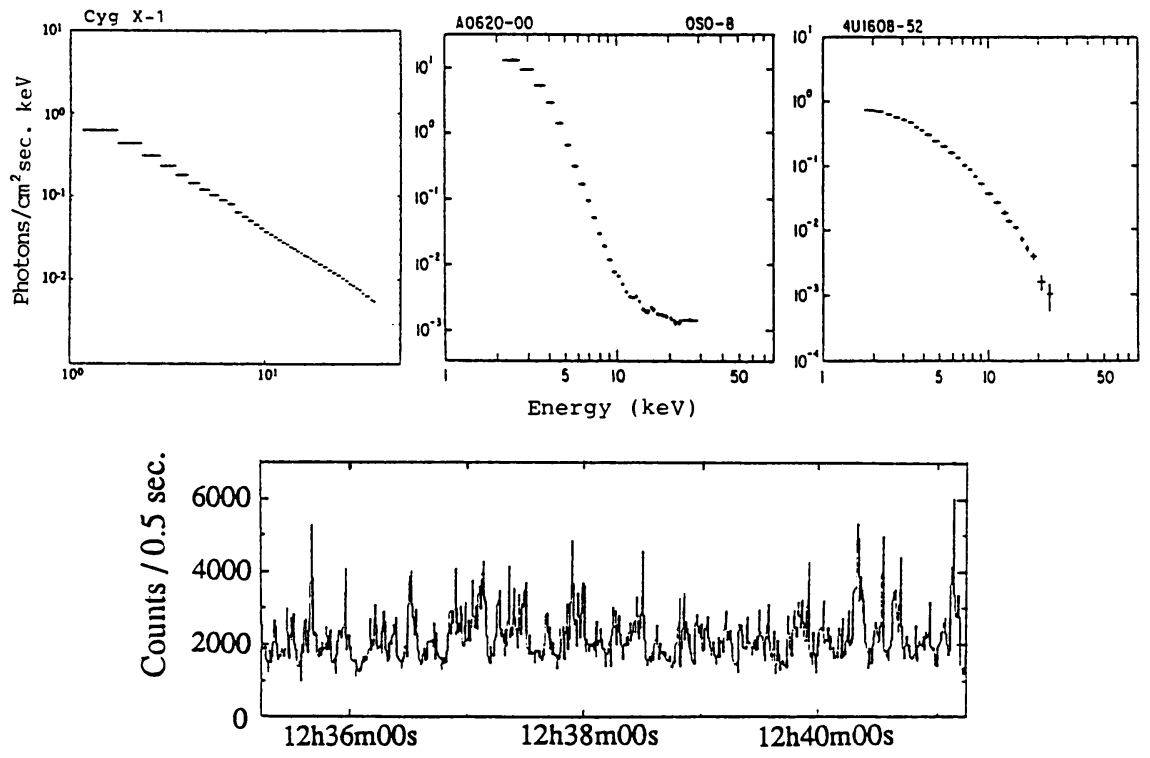

Fig. 7. Energy spectra of the black hole candidates, Cyg X-1 (left) and A0620-00 (center), and the neutron star low-mass binary X160852 (right). Flickering of $\mathrm{Cyg} \mathrm{X-1}$ is also shown (lower panel).

in addition to LMC X-1 and LMC X-3 in the Large Magellanic Cloud. However, we suspect that there are many black hole binaries in our galaxy which manifest themselves only occasionally as transient sources.

In addition to those listed in Table 1, several of the previously recorded bright X-ray transients showed an ultrasoft spectrum with a hard tail, characteristic of A0620-00. These are, for instance, 4U163047, H1743-32, H1705-25 (Nova Oph), 4U1543-47 (see White et al. 1984), and GS1454-64 (Kitamoto et al. 1990). Because of the distinct nature of their spectra, we consider that these sources may also be included in the list of black hole candidates.

As a matter of fact, the rate of the transient outburst of such black hole candidates is remarkably high; ten in less than twenty years. Based on these statistics, we shall attempt to estimate, though very crudely, how many black hole binaries exist in our galaxy. For this estimation, we need additional quantities; the recurrence periods of these transients, the efficiency of detection (time and sky coverages, and distances), of which the recurrence periods are of the largest uncertainty. Among those transient black hole candidates, 4U1630-47 is known to be recurrent with a period of $\sim 600$ days, but the recurrence periods of other sources are yet unknown. Considering the time and sky coverages with various satellites in the past, an average recurrence period less than ten years seems unlikely. On the other hand, at least two of the transients had previously been sighted as the optical novae; A0620-00 in 1917 and GS2023+33 in 1938, and possible intermediate outbursts may have been missed. Therefore, it may be a fair assumption for the average recurrence period to lie between 10 and 50 years. 
As a result, the total number of black holes in binaries in our galaxy is estimated to be at least 100 and could well be more than 1000 (Tanaka 1991). Thus, black hole candidates in binaries are likely to be at least as numerous as neutron stars in binaries.

An outstanding aspect is that, for seven transient black hole candidates of which their optical counterparts were identified, all of them are found to be low-mass stars. This fact leads us to an important issue regarding the origin of the black hole low-mass binaries. Obviously, this is closely related to the problem of the origin of the neutron star low-mass binaries. As it appears plausible, essentially the same process would be responsible for the formations of a black hole low-mass binary and a neutron star low-mass binary. If so, a possible scenario of the accretion induced collapse which is proposed for of a nuetron star low-mass binary formation would not work for producing a black hole of $>3 \mathrm{M}_{\odot}$ (note that at least A0620-00 is a secure cases). An already difficult problem has become even more difficult.

\section{REFERENCES .}

Baym,G. and Pethick,C. (1979), Ann. Rev. Astron. Astrophys., 17,415.

Ebisuzaki,T. (1987), Publ. Astron. Soc. Japan, 39, 287.

Elvis,M. et al. ((1975), Nature, 257, 656.

Inoue, H. et al. (1984), Publ. Astron. Soc. Japan, 36, 831.

Inoue, H. (1986), Proc. IAU Symp. No.125 on "The Origin and Evolution of Neutron Stars", Nanjin, 1986.

Kaluzienski,L.J. et al. (1977), Astrophys. J., 212, 203.

Kitamoto, S. et al. (1990), Astrophys. J., 361, 590.

Lewin,W.H.G. and Joss,P.C. (1983), Accretion-Driven Stellar X-Ray Sources, eds. W.H.G.Lewin and E.P.J.van den Heuvel (Cambridge University Press), p41.

London,R.A., Taam,R.E. and Howard, W.M. (1986), Astrophys. J. 306,142.

Lund,N. and Brandt,S. (1991), IAU Circular 5161.

Magnier,E. et al. (1989), M.N.R.A.S., 237, 729.

Makino,F. (1989), IAU Circular 4782, and IAU Circular 5161.

McClintock,J.E. (1990), Proc. Texas-ESO-CERN Symp. on Relativistic Astrophysics, 1990 (N.Y. Academy of Sciences).

Nagase,F. (1989), Publ. Astron. Soc. Japan, 41, 1.

Nagase,F. (1989), Proc. 23rd. ESLAB Symp. (ESA SP-296), p45.

Nakamura, N., Inoue,H. and Tanaka,Y. (1988), Publ. Astron. Soc. Japan, 40, 209 .

Tanaka,Y. (1986), Proc. IAU Symp. No.125 on "The Origin and Evolution of Neutron Stars", Nanjin, 1986.

Tanaka,Y. (1989), Proc. 23rd. ESLAB Symp. (ESA Sp-296), p3.

Tanaka,Y. (1991), Proc. Symp. on "X-Ray Binaries and the Formation of Binary and Millisecond Radio Pulsars", Santa Barbara, 1991.

Tawara,Y. et al. (1985), Nature, 318, 545.

Tsunemi,H. et al. (1989), Astrophys. J. Letters, 337, L81.

Waki,I. et al. (1984), Publ. Astron. Soc. Japan, 36, 819.

White,N.E., Kaluzienski,J.L. and Swank,J.H. (1984), High Energy

Transients in Astrophysics, ed. S.E.Woosley (N. Y.: AIP), p31. 